\title{
A STUDY OF INTER- AND INTRALINGUAL ASSOCIATIONS IN ENGLISH AND GERMAN ${ }^{1}$
}

\author{
Klaus F. Riegel and Irina W. M. Zivian \\ University of Michigan
}

\begin{abstract}
Free associations and eight types of restricted associations were obtained to 40 nouns from 24 American students of German under four conditions: EE, GG, EG and GE (where English (E) and German (G) are stimulus and response languages, respectively). Because many interlingual responses were translations or partially identical with the stimuli their interlingual variability was lower than that of the intralingual free associations. Interlingual responses were primarily, paradigmatic whereas intralingual responses were syntagmatic. The variabilities of all interlingual and of some intralingual restricted associations were larger than those of free associations. The overlap between different types of restricted associations was markedly higher for the second than for the first language. In the interpretations, classes of possible responses were compared with "subjective" response preferences and sets.
\end{abstract}

Psychologists have given increasing attention to problems of second language learning and bilingualism (for recent reviews see McNamara 1967; Jakobovits 1969, 1970). However, few studies have explored the psycholinguistic process involved. Instead, they have emphasized individual differences in performance. The present study, while elaborating some of the earlier results, is specifically related to a model of bilingual development proposed by the present author (Riegel 1968). Its predictions are derived from the notion that response variability (as well as response speed) are determined, on one hand, by the number of items available in a repertoire. If this number is small, as for second language learners, the number of different associations will also be small. On the other hand, response variability (and speed) will be determined by specific response preferences or sets. If, for instance, second language learners prefer translation responses under interlingual conditions, their response variability will be further reduced.

Both general propositions, the first emphasizing the objective

1This study was supported by Grant 1 P01 HD 01368 from the National Institute of Child Health and Human Development, USPHS. The assistance of Dr. Ruth M. Riegel and Mr. P. R. Kingsley is gratefully acknowledged. 
possibilities for responding and the second, subjective response preferences and sets, interact under various conditions. Whenever the first is emphasized, a specific hypothesis can be derived. Whenever the second is emphasized an alternative hypothes is is available. In the present study response variability but not response speed will be investigated and the following four hypotheses and their alternatives will be tested.

(1a) Since the second language vocabulary of our $S s$ is smaller than their first language vocabulary (supporting evidence will be presented), also the set of intralingual relations will be smaller in the second language. (1b) Since specific response preferences or sets are not as firmly established in the second language, words might be connected in a greater number of different ways.

(2a) Because of the differences in the size of the vocabularies, the sets of interlingual relations will fall between the sets of intralingual relations of the first and of the second language. (2b) Under interlingual conditions, second language learners might choose specific response sets, e.g. might (i) select responses partially identical with their stimuli (identical initials), (ii) search for translations or (iii) for substitutions of the stimuli (paradigmatic responses). These tendencies will reduce the response variability under interlingual conditions. Under intralingual conditions, responses will be scattered more randomly. If they can be categorized at all, they are more likely to be of sequential types (syntagmatic responses).

(3a) If we impose additional constraints upon S's responding by asking him to associate within specific semantic or syntactic categories, the response variability for both the first and the second language will decrease. (3b) Since (as implied under hypothesis 1b) stimulus-response relations are insufficiently established in the second language (except for translative tendencies under interlingual conditions), this effect might be reversed. Subsequently, we expect for all but the intralingual condition of English (i) greater response variability for restricted than for free associations, (ii) greater response overlap, i.e. less differentiation of the responding to the same stimuli but under different restrictions, and (iii) greater similarity between restricted and free associative responses.

(4a) Since a person's active vocabulary is smaller than his passive vocabulary, low response variability is more likely to be found in comparisons between the response than the stimulus languages. Because of the limitations in the vocabulary this effect will be strongest if the second language is the response language. It will be of intermediate magnitude for the interlingual conditions and weakest if the first language is the response language. (4b) For 
the alternative hypothesis we would have to assume that a person's active vocabulary is larger than his passive vocabulary and, subsequently, that the order in which the conditions are effected would be reversed. Such an assumption seems less reasonable than those leading to the first three alternative hypotheses. It merely implies a negation of the fourth hypothesis as originally stated.

\section{METHOD}

\section{Subjects}

Ten male and 14 female undergraduates at the University of Michigan participated in the experiment. Subjects had between one and five years of formal training in German at the high school or college level. Sixteen of the S's had between one and thirteen months of experience in a German speaking country. All were volunteers and were paid for their services.

\section{Materials and Procedure}

When Ss signed up for the experiment, two verbal fluency tests were administered in a counterbalanced order. Subjects were asked to write down as many English (or German) words as they could think of during two 3-minute periods. The verbal fluency tests which do not represent a central part of the experiment) provide estimates of $S s^{\prime}$ active vocabularies. On the average, $S$ s listed 66 English and 31 German words per 3-minute interval. Since there was no overlap in the numbers of words listed in the two languages, this difference is highly significant and supports the assumptions implied in the hypotheses 1 and 2 .

The word association tests which provide the main body of our data were administered individually with self-explanatory instructions. A test consists of five free and four sets of eight restricted association tasks. Free associations were always given between two and four days prior to the restricted associations because performance on the latter may influence that on the former while the reverse is not likely. The four sets of restricted associations were administered in two sessions between two and four days apart from one another.

The following 40 pairs on noun-equivalents were taken from the Michigan norms (Riegel, 1965ab; Zivian, Riegel, \& Zeiher 1967) and were used as stimuli for both the free and the restricted association tasks. The stimuli were presented in two serial orders. The orders were randomly selected for each task. However, both orders were used an equal number of times for each type of re- 
stricted and for the free association task and for each testing condition. Fourteen of the English words occur more than 800 times (Thorndike \& Lorge 1944); thirteen words occur between 200 and 800 times, and thirteen words occur less than 200 times.

High frequency words: arm - Arm, bed - Bett, bird - Vogel,
body - Körper, boy - Junge, door - Tür, Food - Nahrung, head -
Kopf, man - Mann, road - Weg, soldier - Soldat, sun - Sonne,
town - Stadt, work - Arbeit.

Medium frequency words: anger - Ärger, building - Gebăude, cave - Hōhle, cheese - Kãse, cottage - Hütte, dream - Traum, furniture - Möbel, justice - Gerechtigkeit, knife - Messer, star Stern, stream - Bach, thief - Dieb, tool - Werkzeug.

Low frequency words: butterfly - Schmetterling, crust - Kruste, foreigner - Ausländer, glue - Leim, leaf - Blatt, lettuce - Salat, patriot - Patriot, scissors - Schere, sex - Geschlecht, socialism Sozialismus, stool - Hocker, stomach - Magen, tiger - Tiger.

Denoting English words in the stimulus-response sequences by E, German words by $G$, and French words by $F$, free associations were obtained from each $S$ under five conditions of which EE, GG, EG, and GE were administered in counterbalanced order. FE was applied for the purpose of comparing responses given to (presumably) unknown stimuli with those to stimuli with which Ss were partially (German stimuli) or fully familiar (English stimuli). It turned out, however, that twelve Ss had some familiarity with French. In order to avoid distortions of the main body of data, FE was always given first. The instructions used for FE were similar to those for the other four conditions:

The following are tasks of free associations. You have received four pages with 40 stimulus words on each page. The stimulus words are either German or English. Write down the first word that comes into your mind after you have read a stimulus word. Your response is to be either an English or a German word, according to the letter that is encircled at the top of the page. Throughout, respond with single words only, and do not omit any items.

Each $S$ also responded under all four conditions, EE, GG, EG, and $\mathrm{GE}$, to the eight restricted association tasks. The order or the conditions was counterbalanced and the order of the eight types of restrictions was randomized for each condition and for each $S$ : 
"The following are tasks of restricted associations. You have received eight pages with 40 stimulus words on each page. On top of each page you will find a particular title. This title denotes the task you are supposed to perform. Again, your response word is supposed to be either a German or an English word, according to the encircled letter at the top of the page. The following are explanations of the tasks:

Superordinates: Find a classname for the stimulus. For instance, classnames for FORK are SILVERWARE or UTENSIL. Classnames for LIMOUSINE are CAR or VEHICLE.

Similars: Find a word that means essentially the same as the stimulus. For instance, a RAKE or BRANCH may be regarded as similars to FORK. AUTO or AUTOMOBILE may be regarded as similars to CAR.

Functions (Verbs): Find a verb that denotes a usage of the stimulus. For instance, a FORK is used to EAT or TAKE-UP, A CAR is used to TRAVEL or DRIVE.

Qualities (Adjectives): Find an adjective that denotes a quality of the stimulus. For instance a FORK is POINTED or HEAVY. A CAR is FAST or SHINY.

Foregoing Words: Find a word that often precedes the stimulus word in a sentence. For example in the sentence "The plumber fixed the copper pipe," PLUMBER, FDXED and COPPER are foregoing words to PIPE.

Following Words: Find a word that often follows the stimulus word in a sentence. For example in the sentence "The pipe belongs to her old father," FATHER, OLD, and BELONGS are following words to PIPE.

Locations: Name a location of the stimulus. For instance, locations of FORK are TABLE or KITCHEN. Locations for a CAR are ROAD or GARAGE.

Parts: Name an essential part or attribute of the stimulus. For instance, essential parts of a FORK are HANDLE or METAL. Essential attributes of WISDOM are EXPERIENCE and MATURITY."

For most forms of analysis the responses given under the four conditions have been combined in all possible pairs. In particular, EE plus GE vs. GG plus EG compares the effect of the response languages. EE plus EG vs. GG plus GE compares the effect of the stimulus languages. EE plus GG vs. GE plus EG compares intralingual with interlingual conditions of responding. In the 
variance analyses applied, the last comparison is represented by the interaction effect. Neither in the free nor in any of the restricted association tasks did the percentages of response omissions exceed $6 \%$. The average number of omissions was $1.6 \%$.

\section{RESULTS AND DISCUSSION}

\section{Free Associations}

In spite of $S S^{\prime}$ smaller vocabulary, the average intralingual response variability (TTR) is larger for German (GG) than for English (EE), and, the average percentage of the primary responses (PPR) is lower (see Table 1). Thus, the few items available in the second language are connected in a greater number of different ways than in the first language for which particular response preferences seem to have been well established (confirming Hyp. 1b). The TTRs do not differ between the two interlingual conditions, GE and EG, but are markedly lower than for both intralingual conditions, GG and $\mathrm{EE}$; the frequencies of the primary responses are higher (confirming Hyp. 2b). An analysis of variance indicates significant effects of the response language, $F(1,78)=7.77, \mathrm{p}<.01$, and the interaction (inter-vs. intralingual comparison), $F(1,78)=$ $3.97, \mathrm{p}<.01$, but not for the stimulus language (supporting Hyp. 4a).

TABLE 1

Results of free associations for five testing conditions ${ }^{2}$

\begin{tabular}{lccccc}
\hline & TTR & PPR & PSI & PTS & PRO \\
\hline EE & .55 & 32 & 7 & 0 & 4 \\
GG & .63 & 27 & 16 & 0 & 6 \\
EG & .32 & 57 & 27 & 56 & 1 \\
GE & .32 & 63 & 39 & 62 & 1 \\
FE (w) & .43 & 28 & 38 & 53 & 0 \\
(n) & .57 & 22 & 68 & 24 & 0 \\
\hline
\end{tabular}

2The letters in the first column refer to the stimulus and response language respectively: $\mathrm{E}=$ English, $\mathrm{G}=$ German, $\mathrm{F}=$ French, $\mathrm{w}=$ with some knowledge of French, $\mathrm{n}=$ with no knowledge of French; TTR $=$ type-token ratios, PPR $=\%$ of primary responses, PSI $=\%$ of responses with the same initials as the stimuli, PTS $=\%$ of responses identical with translations of stimuli, PRO $=\%$ of response ommissions. 
In exploring the particular response preferences or sets that led to a confirmation of hypothesis $2 b$ under interlingual conditions three methods were applied.

(i) We enumerated the percentages of responses with initials identical with those of their stimuli (PSI). These percentages are lowest for the condition most familiar to Ss, namely for EE (7\%). (This result was replicated when the responses to the same 40 stimuli obtained from the $100 \mathrm{Ss}$ of the Michigan norms were analyzed. In this case PSI equals 9\%.) PSI is much higher for all other and, in particular, for the interlingual conditions. When only those Ss are considered who did not indicate any knowledge of French (n), 68\% of the responses for FE can be accounted for on this basis alone. Subjects with some knowledge of French produce about as many responses of this kind as Ss under the GE condition.

(ii) As the comparisons in PSI between FE and the four conditions show, the strategy of producing responses identical with the stimuli in their initials becomes less important with increasing language proficiency. Subjects sufficiently acquainted with both languages seem to shift to highly organized and selective, namely to translation responses. For an exploration of this proposition, and applying the most stringent criterion of equivalence, we enumerated the percentages with which our own translations of the stimuli (see methods and procedures) were emitted (PTS). Even though these percentages may be somewhat inflated since $S$ s had to go through the lists of equivalent stimuli repeatedly, and thus, may have learned to equate the translations with one another, they are high enough to warrant unambiguous interpretations.

PTS decreases with stimulus frequencies. The 14 most common words produce $60 \%$ translations in the EG conditions, the thirteen medium frequency words produce $55 \%$, and the thirteen low frequency words produce $47 \%$. Similar to PTS, there is a decline with stimulus frequency in PSI from $60 \%$ to $55 \%$ to $44 \%$. All these percentages decline somewhat less regularly for the GE and FE conditions, most likely because the application of the American word count is less appropriate for the German and French stimulusequivalents.

Even though translation seems to be the primary mode of responding under the interlingual conditions, it would be false to regard responding by partial identity, i.e. identity of stimulus and response initials, as a secondary strategy and, possibly, to consider the two percentages as additive. Rather both strategies are interrelated. Among our own translations PSI equals 30\% for the English-German, 32\% for the English-French, and 18\% for the German-French equivalents. 

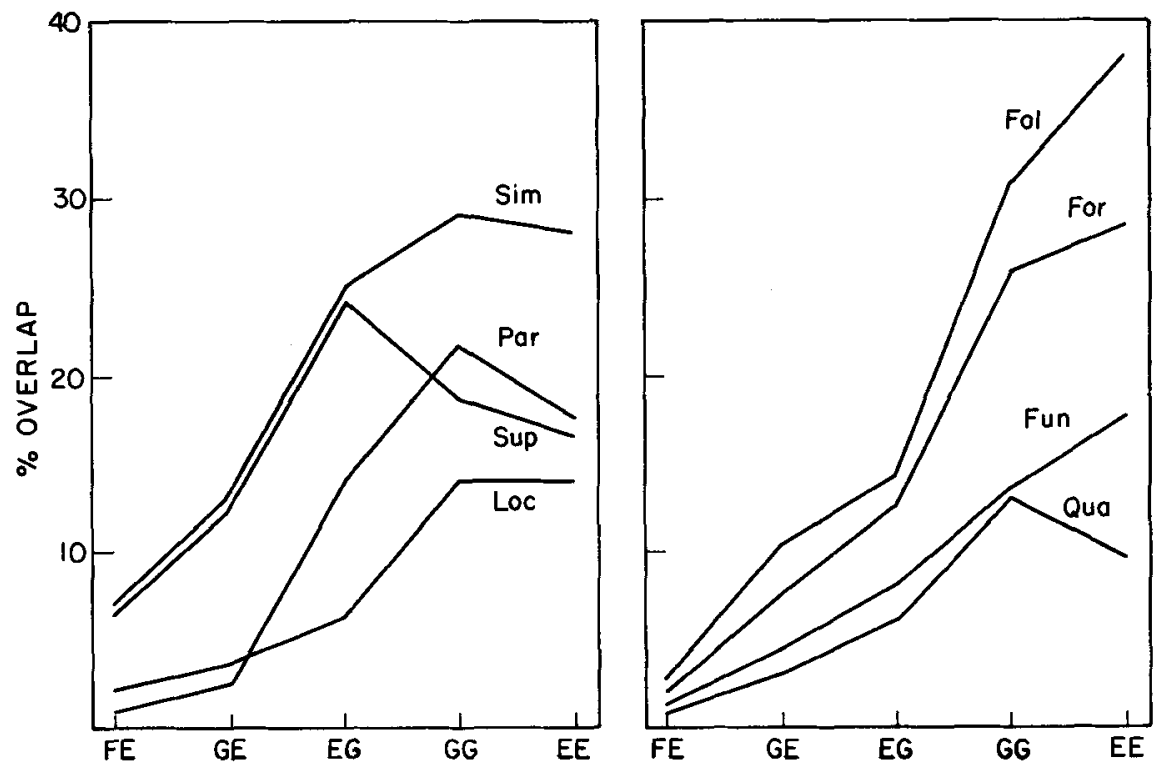

Figure 1. Percent overlaps between free associations and eight types of restricted associations for five testing conditions.

(iii) According to our results $S$ s are not "really free" when responding in a free association task. Under the interlingual conditions in particular, they tend either to translate the stimuli or utilize formal clues to facilitate their performance. The restricted association tasks allow for the analvsis of further response preferences or sets which direct Ss' free associative behavior. For this purpose we compared the eight types of restricted associations of the intralingual conditions (GG and EE) with the five conditions of free associations. In particular, we enumerated the sums of those free associative responses which were identical with the restricted responses given to the same stimuli by our 24 Ss.

In comparing the response languages, more of the German free associations (EG and GG) were accounted for by German restricted associative responses (GG) than were the English free associations (GE and EE) by the English restricted associative responses (EE). The difference averaged over the eight types of restrictions (tasks) was $4 \%, F(1,78)=12.84, p<.01$. In comparing the stimulus languages, the restricted associations accounted for larger percentages of free associations elicited by English than by German stimuli, but these differences were not significant. By far the largest differences were observed for the intra- and interlingual comparison, $F(1,78)=135.18, p<.01$. The results were up to $25 \%$ higher for 
the intralingual condition. The following details are derived from Figure 1.

The largest number of intralingual free associations are accounted for by the syntagmatic responses of Following Words (36\%) and Foregoing Words (30\%). Third in rank are Similars (29\%). For the interlingual conditions, however, Similars and Superordinates account for more free associative responses (19\% and $18 \%$, respectively) than either Following or Foregoing Words (11\% each). Thus, under interlingual conditions $S$ s exhibit stronger tendencies to search for substitutes of the stimuli (paradigmatic responses) rather than to rely on responses that reflect their experience with continuous linguistic discourse (syntagmatic responses). This reresult supplements the previous observation of high translative tendencies under interlingual conditions.

When the number of translations are added to the number of free associations accounted for by the criterion lists of restricted associations (note, translations of the stimuli can not enter into our intralingual criterion lists) the total number of free associations given under EG which can be accounted for (1591) approaches closely that of the responses accounted for under GG (1615). For both GE (1102) and FE (609), however, the figures remain far below that obtained for $\mathrm{EE}$ (1643). ${ }^{3}$ Thus, for GE and FE relatively large numbers of free association responses are neither translations of the stimuli nor can they be accounted for by the restricted associations, but are random variations not determined by any detectable response preferences or sets.

\section{Restricted Associations}

As a comparison of Tables 1 and 2 shows, the TTRs for restricted associations vary less markedly between the four conditions than those for free associations. An analysis of variance of the restricted associations indicates significant effects of the response languages, $F(1,78)=4.10, \mathrm{p}<.01$, and of the interaction (inter - vs. intralingual comparison), $F(1,78)=225.99, p<.01$, but not of the stimulus languages (supporting Hyp. 4a).

When comparisons are made within the interlingual conditions of $E G$ and GE, Ss react always more "freely" on the tasks of restricted than of free associations, i.e. the TTRs for restricted

${ }^{3}$ Four of these five figures are larger than the total number of free associations given under any of the five conditions, i.e. larger than 960 (24 Ss responding to 40 stimuli). Thus the free associations are "overdetermined," i.e. more than one task of restricted associations elicits the same responses to particular stimuli that are identical with those of the free association task. In other words, this observation indicates considerable response overlap between the eight types of restricted associations, an issue to which we shall return further below. 
TABLE 2

Results of restricted associations for four testing conditions averaged over eight tasks 4

\begin{tabular}{lccccc}
\hline & TTR & PPR & PFO & PTO & PRO \\
\hline EE & .50 & 34 & 21 & 20 & 0 \\
GG & .55 & 30 & 21 & 30 & 3 \\
EG & .54 & 30 & 14 & 27 & 2 \\
GE & .54 & 30 & 7 & 20 & 1 \\
\hline
\end{tabular}

associations are always higher (.41 to .76 for EG; .42 to .73 for $\mathrm{GE})$ than those for free associations (.32 for both EG and GE) (supporting Hyp. 3b). This result can be explained by $S s^{\prime}$ translative tendencies in free associations which reduce the variability. For intralingual conditions, Foregoing and Following Words elicit greater TTRs (.72 and .69 for EE; .76 and .70 for GG) than free associations (.55 for EE; .63 for GG) (partially supporting Hyp. $3 b)$. Thus, Ss often react more "freely" under restricted than under free associative instructions and it is questionable whether the latter provide "the most nearly context-free of all the techniques of eliciting verbal responses to particular stimuli" (Deese, 1965 , p. 42).

Our analysis of response preferences and sets in free associations (see preceding section) could provide misleading interpretations if we were to regard all restricted associations as independent. For an analysis of their correlation we enumerated the overlap between the different restrictions separately for each $S$. The overlapping (identical) responses given under any two of the eight restrictions added over stimuli and $S$ s thus were obtained separately. However for more comprehensive comparisons, we derived the sums of overlaps of each restriction with the other seven. Generally, these comparisons reveal that under all conditions Parts, Locations, Similars and Functions are most clearly separated from the other tasks. Following and Foregoing Words are conceptually least distinct.

When conditions are pooled by response languages, German responses are always more overlapping than English responses, $F(1,71)=30.76, \mathrm{p}<.01$, (supporting Hyp. 4a). When the conditions

4See footnote 2(Table 1) for index to most of the letter abbreviations here; in addition, note: PFO $=\%$ of free associative overlaps, PTO $=\%$ of overlaps of any one task of restricted associations with the other seven. 
are pooled by stimulus languages, no significant differences are detected. With the exception of Superordinates and Similars, this holds also for the comparison of intralingual and interlingual conditions.

\section{CONCLUSIONS}

Specific hypotheses about the psycholinguistic behavior of second language learners have been derived from two general propositions. First, response variability will be determined by the responses availäble in a repertoire. Second, response variability will be determined by "subjective" response preferences and sets.

According to our results, the second factor is the more important determinant of Ss' bilingual behavior. Thus, when the TTRs of the free associations were compared between the intralingual conditions (GG vs. EE), the response variability was greater for German than for English even though the differences in vocabulary point in the opposite direction. Presumably, response preferences and sets are insufficiently established for the German language of our $S S$ (Hypothesis 1b). However, response variability is markedly lower for both interlingual than for the intralingual conditions because Ss systematically apply either formal clues (producing responses which have the same initials as the stimuli), translate, or search for substitutes of the stimuli (paradigmatic responses) (Hypothesis 2b). Again, the differences in the vacabulary between the first and second languages would have led to the wrong prediction, namely that response variability under interlingual conditions ought to fall between those of the intralingual conditions of the first and the second language.

When comparing the TTRs of the restricted and the free associations, we should expect lower response variability for the former. Our results indicated, however, that particular response classes and stimulus-response relations do not seem to have been sufficiently established in the second language of our Ss. All TTRs for the interlingual conditions of EG and GE are larger for the restricted than for the free associations. Under the intralingual conditions two tasks of restricted associations (Foregoing Words and Following Words) produce larger response variabilities than free associations (Hypothesis $3 b$ ). These results raise the general question on how free $S S$ actually are when responding in a free association test and with what kind of response preferences and sets they arrive in the laboratory. Even though the results shown in Figure 1 provide some specific answers, this problem has not been pursued much further in the present study. 
Since a person's active vocabulary is smaller than his passive vocabulary, greater differences in response variability were found when the response languages (EE plus GE vs. GG plus EG) rather than the stimulus languages (EE plus EG vs. GG plus GE) were compared (Hypothesis 4a). However, the differences were not in the expected direction; the variability of second language responses was larger than that of the first language. Apparently, Ss connect the few items of their second language in a greater number of different ways than the many items of their first language.

A successful application of the dualistic notion of response availability and "subjective" response preferences and sets is not limited to the study of bilingual behavior. Previously, psycholinguistic performance of creative persons has been characterized by increased response variability in conjunction with well developed response preferences and sets that allow for appropriate and efficient response selections (Riegel, Riegel \& Levine 1966). Psycholinguistic performance of schizophrenics can be characterized by the concepts of overreaction and counterreaction: whenever the external constraint is weak, as under free associations, schizophrenics impose their own, severe response restrictions; whenever the external constraint is strong, as under certain restricted associations, they react very "freely" (Stern \& Riegel 1970). First language acquisition consists in an increase in response variability and in the development of specific response classes, both in regard to language production (Riegel, Riegel, Quarterman \& Smith 1968) and language comprehension (Quarterman \& Riegel 1968; Zivian \& Riegel 1969). Aging leads toward further increases in response variability, primarily caused by a shift toward subjective response sets and by a strong temporary preference for any kind of logical relations at the expense of the more common infralogical and grammatical relations (Riegel \& Riegel 1964, 1968).

The mechanisms of response preferences and sets as applied in the present paper are not vague, mentalistic constructs but have an "objective" basis in the semantic-syntactic structure of the languages as well as in the physical order of the environment with which individuals are confronted. During recent years the study of linguistic structures has attracted much attention. If linguists and psychologists succeed in describing these structures in a comprehensive manner, it will become possible to analyze response preferences and sets "objectively" and to develop a firmer basis for the study of individual and developmental differences. Bilingual behavior, in this respect, does not only represent a specific dimension of variation but also enables us to study the interaction of different linguistic structures, and, perhaps, of some 
invariant features of cognitive-linguistic organization. A comprehensive analysis of these problems may suggest new approaches to second language learning and teaching.

\section{REFERENCES}

Jakobovits, L. A. 1970. Foreign Language Learning. Rowley, Mass.: Newbury House Publishers.

Jakobovits, L. A. 1969. The physiology and psychology of second language learning. In E. Birkmaier (ed.), Britannica Review of Foreign Language Education. Chicago: Encyclopedia Britannica.

McNamara, J. 1967. The bilingual's linguistic performance: a psychological overview. Journal of Social Issues 23. 58-77.

Quarterman, C. J. and K. F. Riegel. 1968. Age differences in the identification of concepts of the natural language. Journal of Experimental Child Psychology 6. 501-509.

Riegel, K. F. 1965a. The Michigan restricted association norms. Report No. 3, USPHS Grant MH 07619, Department of Psychology, University of Michigan.

Riegel, K. F. 1965b. Free associative responses to the 200 stimuli of the Michigan restricted association norms. Report No. 8, USPHS Grant MH 07619, Department of Psychology, University of Michigan.

Riegel, K. F. 1968. Some theoretical considerations of bilingual development. Psychological Bulletin 70, 647-670.

Riegel, K. F. and R. M. Riegel. 1964. Changes in associative behavior during later years of life: a cross-sectional analysis. Vita Humana $7.1-32$.

Riegel, K. F., R. M. Riegel, and R. S. Levine. 1966. An analysis of associative behavior and creativity. Journal of Personality and Social Psychology 4. 50-56.

Riegel, K. F., R. M. Riegel, C. J. Quarterman, and H. E. Smith. 1968. Developmental differences in word meaning and semantic structure. Human Development 11. 92-106.

Stern, E. J. and K. F. Riegel. 1970. Comparisons of the restricted associations of chronic schizophrenic and normal control subjects. Journal of Abnormal Psychology 75. 164-171.

Thorndike, E. L. and I. Lorge. 1944. The Teacher's Word Book of 30,000 Words, New York: Teachers College, Columbia University.

Zivian, M. T. and K. F. Riegel. 1969. Word identification as a function of semantic clues and association strength. Journal of Experimental Psychology 79. 336-341.

Zivian, M. T., K. F. Riegel, and H. Zeiher. 1967. Restricted and free associations of 12,15, and 18 year old Germans. Report No. 29, USPHS Grant HD 01368, Center for Human Growth and Development, University of Michigar. 